\title{
Photoluminescence Study on O-plasma Treated ZnO Thin Films
}

\author{
Jaewon Cho ${ }^{1 *}$, Jinsung Choi ${ }^{1}$, SeGi $\mathrm{Yu}^{2}$, and Seuk Joo Rhee ${ }^{2}$ \\ ${ }^{1}$ Department of Electrophysics, Kwangwoon University, Seoul 139-701, Korea \\ ${ }^{2}$ Department of Physics, Hankuk University of Foreign Studies, Yongin 449-791, Korea
}

(Received August 20, 2013 : revised October 16, 2013 : accepted October 16, 2013)

\begin{abstract}
A temperature dependent (10K-290K) photoluminescence (PL) study for two differently prepared $\mathrm{ZnO}$ thin films (as-grown and O-plasma treated) is presented. Four characteristic peaks were identified for both samples: (i) neutral donor-bound excitons $\left(\mathrm{D}^{\circ} \mathrm{X}\right)$, (ii) two electron satellites (TES), (iii) phonon replica of $\mathrm{D}^{\circ} \mathrm{X}\left(\mathrm{D}^{\circ} \mathrm{X}-1 \mathrm{LO}\right)$, and (iv) donor-acceptor pair transition (DAP). As the sample temperature increased, $\mathrm{D}^{\circ} \mathrm{X}-1 \mathrm{LO}$ and DAP transitions became indistinct. This was accompanied by newly-rising emission of free electron-acceptor transitions $\left(\mathrm{e}, \mathrm{A}^{\circ}\right)$. The spectral evolution with temperature for as-grown samples also showed the optical emission from free excitons, which became dominant at higher temperatures. Some features related to O-plasma were identified in PL spectra: (i) different positions of TES transitions $(28 \mathrm{meV}$ lower than $\mathrm{D}^{\circ} \mathrm{X}$ for as-grown samples and $35 \mathrm{meV}$ for O-plasma treated samples), (ii) the decrease of spectral intensity in both emissions of $\mathrm{D}^{\circ} \mathrm{X}$ and DAP after O-plasma treatment, and (iii) no noticeable transition from free excitons after the O-plasma treatment.
\end{abstract}

Keywords : $\mathrm{ZnO}$ thin film, O-plasma treatment, Photoluminescence spectroscopy

OCIS codes : (300.0300) Spectroscopy; (160.6000) Semiconductor materials; (250.5230) Photoluminescence;

(310.3840) Materials and process characterization

\section{INTRODUCTION}

$\mathrm{ZnO}$ is a semiconductor with direct band structure (band gap energy of $3.37 \mathrm{eV}$ at room temperature), which is similar in physical characteristics to GaN. Carrier doping is the first barrier to be overcome for the application of $\mathrm{ZnO}$ as electronic and photonic devices. While n-type doping can be easily realized to the level up to $10^{21} \mathrm{~cm}^{-3}$ [1], reports on successful p-type doping are rare but gradually increasing [2, 3]. Both bipolar (n-and p-type) doping and wide band gap could maximize the possibility of developing $\mathrm{ZnO}$ as transparent electronic devices and light emitting devices from visible to uv regions[4-7]. In addition, the high exciton binding energy of $\mathrm{ZnO} \quad(\sim 60$ $\mathrm{meV}$, cf. $\sim 25 \mathrm{meV}$ for $\mathrm{GaN}$ ) allows near-band-edge excitonic emission at room and higher temperatures $[5,6,8,9]$. Recently, the technology enabling us to exploit $\mathrm{ZnO}$ is developing fast, however, the fundamental understanding for optical characteristics is still under debate. Besides such a physical understanding, various physical and chemical treatment processes should be linked in order to realize promising devices of $\mathrm{ZnO}$.

Photoluminescence (PL) investigation is a suitable tool to determine the crystal quality and the presence of impurities in the material. In addition, the study at low temperatures gives more detailed comprehension of the physical properties of the sample. In this article, we studied the effects of O-plasma treatment on the physical characteristics of $\mathrm{ZnO}$ thin films with PL spectroscopy, varying sample temperatures from $10 \mathrm{~K}$ to $290 \mathrm{~K}$.

\section{EXPERIMENTAL}

The sample used in this experiment was $\mathrm{ZnO}$ thin films of thickness $\sim 350 \mathrm{~nm}$ grown on c-plane sapphire at $650^{\circ} \mathrm{C}$ by rf-magnetron sputtering in an $\mathrm{Ar}+\mathrm{O}_{2}$ ambient atmosphere. After chemical cleaning, the sample was showered at $300^{\circ} \mathrm{C}$ in O-plasma generated by an RF plasma system. The experimental conditions for the plasma treatment are shown in the Table 1. PL measurement was performed as the sample was kept in the closed cycle cryostat. The

\footnotetext{
*Corresponding author: jcho@kw.ac.kr

Color versions of one or more of the figures in this paper are available online.
} 
TABLE 1. Experimental conditions for the O-plasma treatment

\begin{tabular}{c|c}
\hline \hline Base Pressure & $<5.0 \times 10^{-6}$ torr \\
\hline Process Pressure & $<2.0 \times 10^{-2}$ torr \\
\hline Reactive Gases & $\begin{array}{c}\text { Oxygen } \\
(99.995 \%)\end{array}$ \\
\hline RF Power & $40 \mathrm{~W}$ \\
\hline Substrate Temperature & $300^{\circ} \mathrm{C}$ \\
\hline Process Time & 60 min \\
\hline Sample & ZnO Thin Film on Sapphire \\
\hline
\end{tabular}

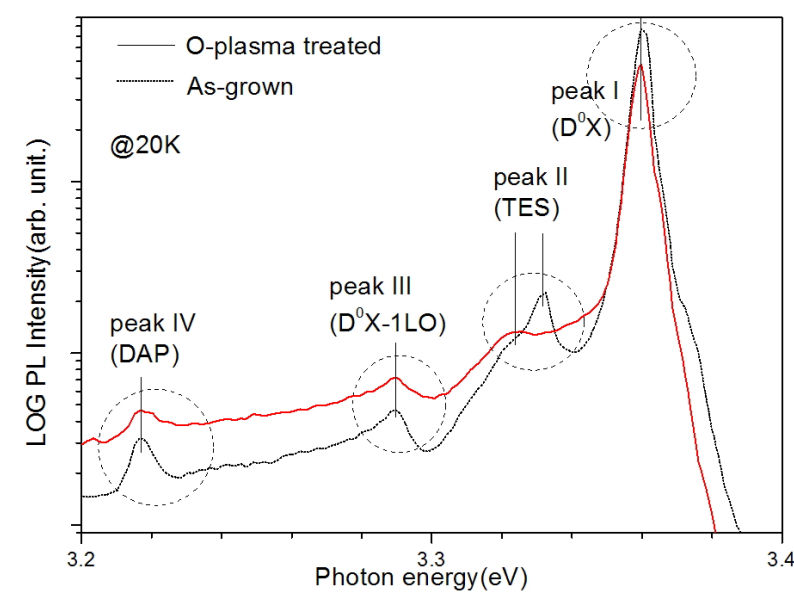

FIG. 1. The comparison of PL spectra from two differently prepared $\mathrm{ZnO}$ samples (as grown and O-plasma treated). The spectra shown in the figure were taken at the sample temperature of $20 \mathrm{~K}$. The PL data are shown on a log intensity scale but are displaced vertically for clarity.

sample temperature was varied from $10 \mathrm{~K}$ to $290 \mathrm{~K}$. The excitation source was a $\mathrm{CW}$ He-Cd laser $(\lambda=325 \mathrm{~nm})$.

\section{RESULTS AND DISCUSSION}

The experimental results of this work are based on the analysis of PL spectra from $\mathrm{ZnO}$ thin films. The spectra were taken at the varying temperatures of the sample from $10 \mathrm{~K}$ to $290 \mathrm{~K}$ from two differently prepared samples, one of which is "as grown" and the other is "O-plasma treated". There was spectral similarity between the two samples, as shown in Fig. 1, where four characteristic peaks (Peak I, II, III and IV) were identified for each sample. Now the physical origins for each peak and the resulting effects of O-plasma treatment on $\mathrm{ZnO}$ films will be examined.

In the undoped bulk $\mathrm{ZnO}$, the neutral shallow donor bound exciton often dominates because of the presence of donors resulting from unintentional impurities and/or shallow donor-like defects [10]. Acceptors like $\mathrm{Na}$ or $\mathrm{Li}$ could be present in $\mathrm{ZnO}$, too [11]. However, the chemical origin and binding energy of the most underlying donor and acceptor atoms remain unclear. It is known that the low-temperature PL spectra are dominated by several bound excitons in the narrow energy range from 3.348 to $3.374 \mathrm{eV}[12]$. The higher energy part of the range is mainly due to the recombination of neutral donor-bound excitons $\left(\mathrm{D}^{0} \mathrm{X}\right)$, while the lower part comes from neutral acceptor bound excitons $\left(\mathrm{A}^{0} \mathrm{X}\right)$ [12]. Peak $\mathrm{I}$ in Fig. 1, appearing at $3.360 \mathrm{eV}$ for both samples (as grown and O-plasma treated), is believed to be mainly the recombination of $\mathrm{D}^{0} \mathrm{X}$, which is in good agreement with the results reported by other groups [12-15].

When $\mathrm{D}^{0} \mathrm{X}$ decays, all the energy of the exciton is converted into the energy of a photon. However, the decay process could involve TES (Two Electron Satellites) transition, where the donor atom is excited to a higher energy state. When this happens, the outcoming photon will have less energy by an amount equal to the energy difference between the first excited and ground states of the donor. For $\mathrm{ZnO}$, the difference is known to be around $30 \mathrm{meV}$, which varies with the donor atoms that localize $\mathrm{D}^{0} \mathrm{X}$ $[12,13,16]$. The TES transition could be identified in our study. Peak II of our spectra depicted in Fig. 1 is considered to be related to the TES transition. Unlike Peak I $\left(D^{0} \mathrm{X}\right)$, however, Peak II appears at different positions according to the sample preparation. The positions are as follows: $28 \mathrm{meV}$ lower than Peak I for as-grown samples and $35 \mathrm{meV}$ for O-plasma treated samples. What this observation implies will be discussed later.

Peak III, appearing at $3.289 \mathrm{eV}$ at $20 \mathrm{~K}$, whose energy is lower than Peak I by about $71 \mathrm{meV}$, is believed to be due to a phonon replica of Peak I, as the longitudinal optical (LO) phonon energy of $\mathrm{ZnO}$ is known to be 71-73 $\mathrm{meV}$ [17].

When there exists both donor and acceptor impurities together, the recombination of an electron and a hole can take place via donor-acceptor pair (DAP) transitions. The DAP transition, which is observed at low temperatures, happens through a quantum tunneling process when impurities are further away from each other than effective Bohr radius. The photon energy for the DAP transition is as follows; $h v=E_{g}-E_{D}-E_{A}+q^{2} / \epsilon r[18]$. The experimental evidence for the DAP transition is found in many other works, and the outgoing photon energy related to the DAP transition is known to be near $3.22 \mathrm{eV}$ for $\mathrm{ZnO}[12,16$, 19, 20]. The DAP transition could be identified in our experiment, too. Peak IV, appearing at $3.217 \mathrm{eV}$ for both samples, is considered to be the emission from the DAP transitions.

Figure 2 and Fig. 3 show the evolution of PL spectra with sample temperatures for O-plasma treated and as-grown samples, respectively. Common features can be found in the both figures. The Peak III and IV, which are distinct in spectra at low temperatures of $10 \mathrm{~K}-30 \mathrm{~K}$, become obscured at higher temperatures. This, instead, accompanies the generation of a new peak positioned at 


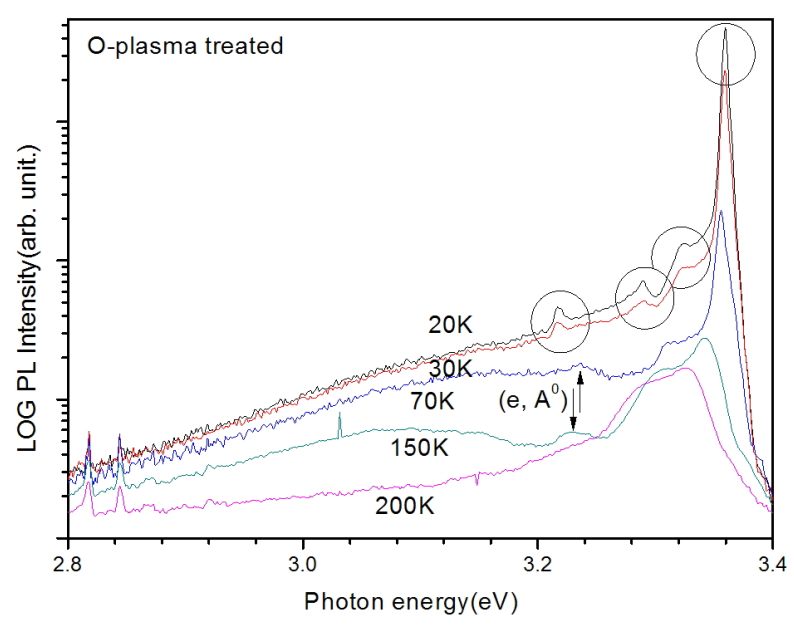

FIG. 2. These PL spectra were selected at several representative temperatures $(20 \mathrm{~K}, 30 \mathrm{~K}, 70 \mathrm{~K}, 150 \mathrm{~K}$ and $200 \mathrm{~K})$ for the O-plasma treated $\mathrm{ZnO}$ film. At $70 \mathrm{~K}$, a new peak (e, $\mathrm{A}^{0}$ ) around $3.24 \mathrm{eV}$ (depicted byarrows) arises while both $\mathrm{D}^{0} \mathrm{X}-1 \mathrm{LO}$ and DAP peaks vanish.

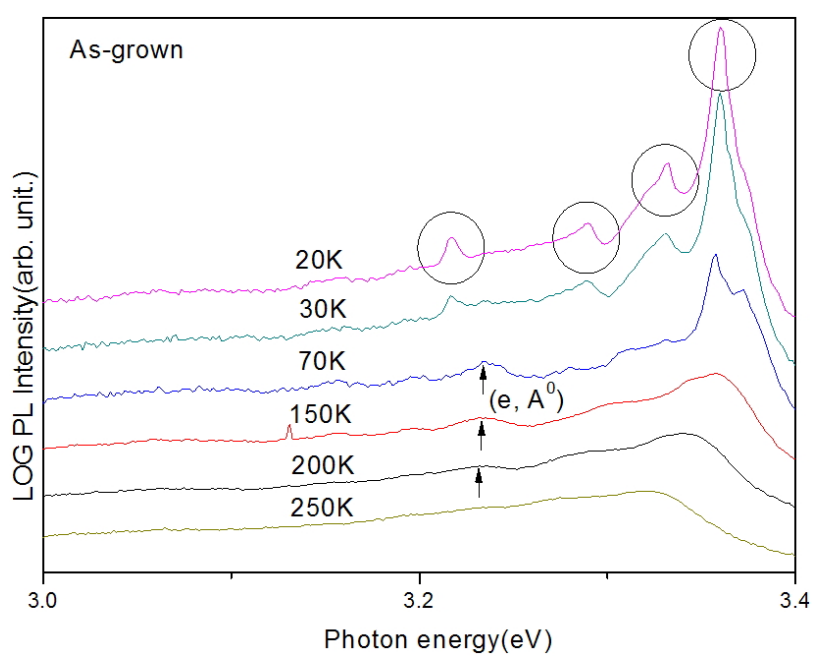

FIG. 3. The PL spectra recorded at different sample temperatures $(20 \mathrm{~K}, 30 \mathrm{~K}, 70 \mathrm{~K}, 150 \mathrm{~K}, 200 \mathrm{~K}$, and $250 \mathrm{~K})$ for the as-grown $\mathrm{ZnO}$ film. The plots are vertically shifted, each for a better visibility. The intensities of $\mathrm{D}^{0} \mathrm{X}-1 \mathrm{LO}$ and DAP peaks decrease and a free electron-acceptor transition peak (e, $\left.\mathrm{A}^{0}\right)$ arises around $3.24 \mathrm{eV}$ at $70 \mathrm{~K}$.

3.23-3.24 eV which is designated by an arrow in the figures. The new peak was distinguishable up to $200 \mathrm{~K}$ for as-grown samples and $180 \mathrm{~K}$ for O-plasma treated samples. This experimental observation indicates the thermal ionization of neutral donors. Donors in $\mathrm{ZnO}$ get thermally ionized more and more as the temperature gets higher. Free electrons from the neutral donors are expected to recombine with the neutral acceptors, which is denoted by the free electronacceptor transition $\left(\mathrm{e}, \mathrm{A}^{0}\right)$. This $\left(\mathrm{e}, \mathrm{A}^{0}\right)$ peak will appear at higher energies than the DAP peak, and similar results were reported in other works $[12,16,19]$. Hence, the

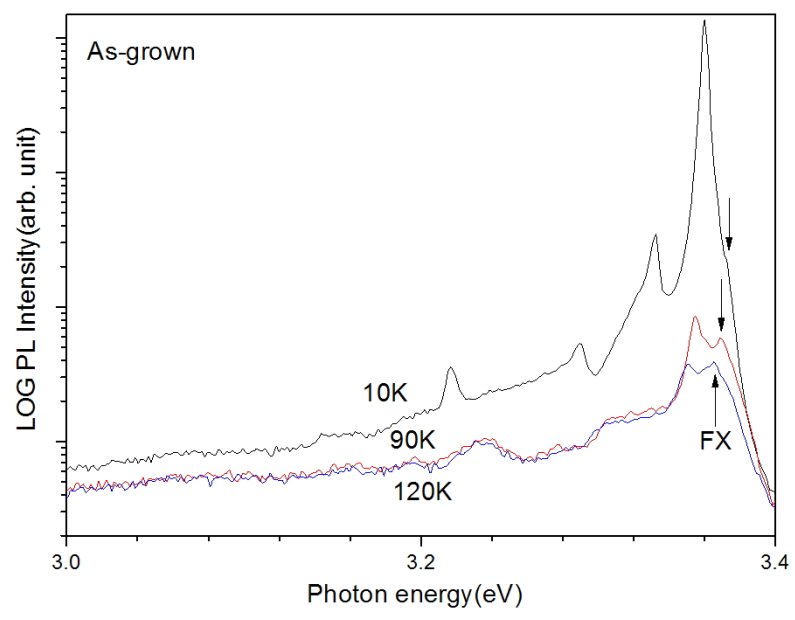

FIG. 4. The changes in the PL spectra with increasing temperature for the as-grown $\mathrm{ZnO}$ film. Transitions due to free excitons are depicted by arrows.

newly-rising peak at $3.23-3.24 \mathrm{eV}$ is believed to be the emission from the $\left(\mathrm{e}, \mathrm{A}^{0}\right)$ transition.

Figure 4 shows spectral evolution with temperatures for the as-grown sample, where a new peak, designated by arrows, could be identified at the right shoulder of Peak I $\left(\mathrm{D}^{0} \mathrm{X}\right)$. The peak gets more distinct as temperature increases. The position of the peak is $3.369 \mathrm{eV}$ at $90 \mathrm{~K}$, which is higher by $14.1 \mathrm{meV}$ than the $\mathrm{D}^{0} \mathrm{X}$ peak. We believe that this peak comes from the decay of the free exciton, considering the relative position of the peak to $\mathrm{D}^{0} \mathrm{X}$ along with characteristic features of temperature evolution. The localization energy of bound excitons in $\mathrm{ZnO}$ is known to be $10-20 \mathrm{meV}[12,14,15,20]$, which is in a good agreement with our result. This indicates that bound excitons change into free excitons as the temperature increases. The free exciton peak gets larger with the increase of the temperature, and starts to surpass the $\mathrm{D}^{0} \mathrm{X}$ peak at $120 \mathrm{~K}$, as shown in the figure. This tendency continues, and free excitons are dominant from $150 \mathrm{~K}$.

Let us consider the effects of O-plasma treatment on the properties of $\mathrm{ZnO}$ film. The plasma treatment process can induce both physical and chemical effects on the sample. The atoms and/or ions in the plasma will penetrate into the sample, damaging the crystal and making chemical bonds with the atoms in the sample. As a result, the plasma process induces crystal defects as well as impurities in the sample. Oxygen atoms and ions in the O-plasma are expected to make chemical bonds with donor-like impurities and defects which are known to exist in an undoped $\mathrm{ZnO}$. They can also fill O-vacancies which are known to play an important role as donors in $\mathrm{ZnO}$ along with interstitial $\mathrm{Zn}$ atoms. As a result, the density of the donor-like defects decreases in the plasma process, which results in decrease of the intensity of $\mathrm{D}^{0} \mathrm{X}$ and DAP transitions. This was identified in our experimental result. Comparing the intensities of the $\mathrm{D}^{0} \mathrm{X}$ peak and the DAP 
peak for both samples at $10 \mathrm{~K}$, we could find that the intensity decreased by about $40 \%$ for the $\mathrm{D}^{0} \mathrm{X}$ peak and $60 \%$ for the DAP peak after the plasma treatment. Oxygen atoms and defects, which are generated in the sample during the plasma process, can become new centers that localize excitons. Some of those centers will act as neutral donors to capture excitons, which results in another $\mathrm{D}^{0} \mathrm{X}$. The difference in the position of Peak II between both samples, as stated above in Fig. 1, can be understood to be due to newly generated neutral donors after the O-plasma treatment.

Another feature, to be noted, is that the remarkable recombination of free excitons was not identified for the O-plasma treated sample. It is difficult to determine the physical origin of the phenomena based solely on our data, but it is suggestive that the higher defect and impurity concentrations induced in the sample by O-plasma can make the formation of free excitons difficult and the lifetime of the free exciton shorter. Hence, the peak from free excitons seems to be shaded after the plasma treatment.

\section{CONCLUSION}

The PL intensity concerning $\mathrm{D}^{\circ} \mathrm{X}$ and DAP decreased after the O-plasma treatment by $40 \%$ and $60 \%$ respectively at $10 \mathrm{~K}$. These two emissions are closely related to neutral donors in $\mathrm{ZnO}$, which indicates that the O-plasma process induces a remarkable decrease of neutral donors. Considering that the $\mathrm{O}$-vacancies and interstitial $\mathrm{Zn}$ atoms play an important role as donors in $\mathrm{ZnO}$, the result implies that oxygen atoms and ions in the O-plasma penetrate into the film, filling O-vacancies and making chemical bonds with interstitial $\mathrm{Zn}$ atoms. And crystal defects and impurities caused during the O-plasma process are expected to become new centers that localize excitons, which is responsible for the different position of TES emissions $(28 \mathrm{meV}$ lower than $\mathrm{D}^{\circ} \mathrm{X}$ for as-grown samples and $35 \mathrm{meV}$ for O-plasma treated samples). In our study, no significant trace of free excitons has been observed in the PL data after the O-plasma treatment. This could be attributed to higher defect and impurity concentrations generated in the sample by the O-plasma treatment.

\section{ACKNOWLEDGMENT}

The present research had been conducted by the Research Grant of Kwangwoon University in 2012.

\section{REFERENCES}

1. K. Ellmer, "Magnetron sputtering of transparent conductive zinc oxide: relation between the sputtering parameters and the electronic properties," J. Phys. D: Appl. Phys. 33,
R17-R32 (2000).

2. D. C. Look, B. Clafin, Y. I. Alivor, and S. J. Park, "The future of $\mathrm{ZnO}$ light emitters," Phys. Stat. Sol. A 201, 2203-2212 (2004).

3. Y. R. Ryu, S. Zhu, D. C. Look, J. M. Wrobel, H. M. Jeong, and H. W. White, "Synthesis of p-type ZnO films," J. Cryst. Growth 216, 330-334 (2000).

4. Y. F. Chen, D. M. Bagnall, H. Koh, K. Park, K. Hiraga, Z. Zhu, and T. Yao, "Plasma assisted molecular beam epitaxy of $\mathrm{ZnO}$ on c-plane sapphire: Growth and characterization,” J. Appl. Phys. 84, 3912-3918 (1998).

5. W. Y. Liang and A. D. Yoffe, "Transmission spectra of ZnO single crystals," Phys. Rev. Lett. 20, 59-62 (1968).

6. D. C. Reynolds, D. C. Look, B. Jogai, C. W. Litton, G. Cantwell, and W. C. Harsch, "Valence-band ordering in ZnO,” Phys. Rev. B 60, 2340-2344 (1999).

7. D. P. Yu, Z. G. Bai, Y. Ding, Q. L. Hang, H. Z. Zhang, J. J. Wang, Y. H. Zou, W. Qian, G. C. Xiong, H. T. Zhou, and S. Q. Feng, "Nanoscale silicon wires synthesized using simple physical evaporation," Appl. Phys. Lett. 72, 3458-3460 (1998).

8. P. Zu, Z. K. Tang, G. K. L. Wong, M. Kawasaki, A. Ohtomo, H. Koinuma, and Y. Segawa, "Ultraviolet spontaneous and stimulated emissions from $\mathrm{ZnO}$ microcrystallite thin films at room temperature," Solid State Commun. 103, 459-463 (1997).

9. D. M. Bagnall, Y. F. Chen, M. Y. Shen, Z. Zhu, T. Goto, and T. Yao, "Room temperature excitonic stimulated emission from zinc oxide epilayers grown by plasmaassisted MBE," J. Cryst. Growth 184/185, 605-609 (1998).

10. Ü. Özgür, Ya. I. Alicov, C. Liu, A. Teke, M. A. Reshchikov, S. Dogan, V. Avrutin, S. J. Cho, and H. Morkoç, "A comprehensive review of $\mathrm{ZnO}$ materials and devices,” J. Appl. Phys. 98, 041301-1 041301-3 (2005).

11. A. Kobayashi, O. F. Sankey, and J. D. Dow, "Deep energy levels of defects in the wurtzite semiconductors AIN, CdS, CdSe, ZnS, and ZnO," Phys. Rev. B 28, 946-956 (1983).

12. A. Teke, Ü. Özgür, S. Dogan, X. Gu, H. Morkoc, B. Nemeth, J. Nause, and H. O. Everitt, "Excitonic fine structure and recombination dynamics in single-crystalline ZnO," Phys. Rev. B 70, 195207-1 195207-10 (2004).

13. H. Alves, D. Pfisterer, A. Zeuner, T. Riemann, J. Christen, D. M. Hofmann, and B. K. Meyor, "Optical investigations on excitons bound to impurities and dislocations in $\mathrm{ZnO}$," Optical Materials 23, 33-37 (2003).

14. D. W. Hamby, D. A. Lucca, M. J. Klopfstein, and G. Cantwell, "Temperature dependent excitation photoluminescence of bulk ZnO," J. Appl. Phys. 93, 3214-3217 (2003).

15. L. Wang and N. C. Giles, "Temperature dependence of the free-exciton transition energy in zinc oxide by photoluminescence excitation spectroscopy," J. Appl. Phys 94, 973-978 (2003).

16. K. Thonke, Th. Gruber, N. Teofilov, R. Schonfelder, A. Wagg, R. Sauer, "Donor-acceptor pair transitions in $\mathrm{ZnO}$ substrate material," Physica B 308-310, 945-948 (2001).

17. T. C. Damen, S. P. Porto, and B. Tell, "Raman effect in Zinc oxide," Phys. Rev. 142, 570-574 (1966).

18. J. I. Pankove, Optical Processes in Semiconductors (Dover Publications, Inc.), p. 143.

19. K. Tamura, T. Makito, A. Tsukazaki, M. sumiya, S. Fuke, 
T. Furumochi, M. Lippmaa, C. H. Chia, Y. Segawa, H. Koinuma, and M. Kawasaki, "Donor-acceptor pair luminescence in nitrogen-doped $\mathrm{ZnO}$ films grown on lattice-matched $\mathrm{ScAlMgO}_{4}$ (0001) substrates," Solid State Communications 127, 265-269 (2003).
20. B. K. Meyer, H. Alves, D. M. Fofmann, W. Kriegseis, D. Forster, F. Bertram, J. Christen, A. Hoffmann, M. Straßburg, M. Dworzak, U. Haboeck, and A. V. Rodina, "Bound exciton and donor-acceptor pair recombinations in ZnO,” Phys. Stat. Sol. (b) 241, 231-260 (2004). 